\title{
Numerical Approach for Differential-Difference Equations having Layer Behaviour with Small or Large Delay using Non-Polynomial Spline
}

M Lalu

Osmania University University College of Engineering

K. Phaneendra

Osmania University University College of Engineering

Siva Prasad Emineni ( $\nabla$ emineni@yahoo.co.in )

Osmania University University College of Engineering https://orcid.org/0000-0002-1253-5569

\section{Research Article}

Keywords: Non-polynomial spline, Differential - difference equation, Layer behaviour, Delay, Fitting parameter, Difference approximation

Posted Date: June 30th, 2021

DOI: https://doi.org/10.21203/rs.3.rs-261904/v1

License: (a) (1) This work is licensed under a Creative Commons Attribution 4.0 International License. Read Full License

Version of Record: A version of this preprint was published at Soft Computing on August 18th, 2021. See the published version at https://doi.org/10.1007/s00500-021-06032-5. 


\title{
Numerical Approach for Differential-Difference Equations having Layer Behaviour with Small or Large Delay using Non-Polynomial Spline
}

\author{
M. Lalu, K. Phaneendra, E. Siva Prasad* \\ Department of Mathematics, University College of Engineering, Osmania University, \\ Hyderabad, India. \\ * Corresponding author, e-mail: emineni@yahoo.co.in
}

\begin{abstract}
A numerical approach is suggested for the layer behaviour differential-difference equations with small and large delays in the differentiated term. Using the non-polynomial spline, the numerical scheme is derived. The discretization equation is constructed using the first order derivative continuity at non-polynomial spline internal mesh points. A fitting parameter is introduced into the scheme with the help of the singular perturbation theory to minimize the error in the solution. The maximum errors in the solution are tabulated to verify the competence of the numerical method relative to the other methods in literature. We also focused on the impact of large delays on the layer behaviour or oscillatory behaviour of solutions using a special mesh with and without fitting parameter in the proposed scheme. Graphs show the effect of the fitting parameter on the solution layer.
\end{abstract}

Keywords. Non-polynomial spline; Differential - difference equation; Layer behaviour; Delay; Fitting parameter; Difference approximation.

2010 Mathematics Subject Classification. 65L10, 65L11, 65L12

\section{Introduction}

Differential- difference equations are problems in which the time evolution of the state variable can depend on specific past in some arbitrary way, i.e., the rate of physical system change depends not only on the state of the physical system but also on its history [1]. Such problems also occur when many practical phenomena are modelled such as thermo-elasticity [2], hybrid optical system [3], in population dynamics [14], red blood cell system [17], in models for physiological processes [18], predator-prey models [19], optimal control theory [7] and in the potential in nerve cells by synaptic inputs in dendrites [26]. Further analysis on 
mathematical aspects of the above group of modelling problems and of the singular perturbations is available from the collection of books to name but a few, Bellman and Cooke [1], Doolan et al. [4], Driver [5], El'sgol'ts and Norkín [6], Kokotovic et al. [13], Miller et al. [20] and Smith [25].

The authors in $[15,16]$ provided asymptotic access for a class of layer behaviour differential equations. In [15], researchers provided a mathematical model of evaluating the expected time to produce potential action in nerve cells with random synaptic inputs in dendrites. The researchers in [16] illustrated the examples with quick oscillations. Kadalbajoo and his team initiated an extensive numerical work using finite differentiation techniques [9, 10, 11, 12]. Ravikanth and Murali [23] have proposed a fitted method to solve the problems through tension splines, which only contains a delay in the differentiated term.

\section{Declaration of the problem}

Consider the equation with a retarded term, i.e., negative shift or delay in a differentiated term

$$
\varepsilon w^{\prime \prime}(s)+P(s) w^{\prime}(s-\delta(\varepsilon))+Q(s) w(s)=R(s), \quad 0<s<1,
$$

subject to the boundary conditions

$$
w(s)=\phi(s),-\delta \leq s \leq 0 ; w(1)=\gamma,
$$

where $0<\varepsilon \ll 1$ is a perturbation parameter, $P(s), Q(s), R(s)$ and $\varphi(s)$ are continuous functions in $[0,1], \gamma$ is a finite constant and $\delta$ is the delay parameter. It is recognized that when $\delta=0$, Eq. (1) is changes to a singularly perturbed equation exhibit layer behaviour and turning points depending upon the component of the respective convention and reaction term. The solution $w(s)$ display layer on the left-end when $P(s)$ is positive or on the right-end when $P(s)$ is negative all through the interval [0 1]. The solution's layer behaviour is no longer preserved when $\delta(\varepsilon)$ is in order $O(\varepsilon)$, and the solution exhibits oscillatory behaviour.

\section{Non-polynomial spline}

With mesh length $h=\frac{1}{N}$, the domain $[0,1]$ into $N$ subdomains, so that $s_{i}=i h, \forall i=0$, $1, \ldots, N$ with $0=s_{0}, 1=s_{N}$. Let $w(s)$ be the exact solution and $w_{i}$ be an approximation to $w(s)$ by the non-polynomial spline $\Psi_{i}(s)$ communicating through the $\left(s_{i}, w_{i}\right)$ and $\left(s_{i+1}, w_{i+1}\right)$. We need not only $\Psi_{i}(s)$ satisfy the interpolatory conditions at $s_{i}$ and $s_{i+1}$, but also to perform the continuity of the first derivative at the common nodes $\left(s_{i}, w_{i}\right)$. The cubic non-polynomial spline $\Psi_{i}(s)$ has the form for each $i^{\text {th }}$ division (Ref. [8])

$\Psi_{i}(s)=A_{i}+B_{i}\left(s-s_{i}\right)+C_{i} \sin \tau\left(s-s_{i}\right)+L_{i} \cos \tau\left(s-s_{i}\right), \forall i=0,1,2, \ldots, N-1$, 
where $A_{i}, B_{i}, C_{i}$ and $L_{i}$ are to be determine and $\tau$ is a free parameter.

The function $\Psi_{i}(s)$ of the class $C^{2}[0,1]$ interpolates $w\left(s_{i}\right)$ at mesh points $s_{i}, \forall i=0,1, \ldots, N$, depending on a parameter $\tau$ and transformed to the usual cubic spline as $\tau \rightarrow 0$.

To deduce the values of the coefficients in Eq. (3) in term of $w_{i}, w_{i+1}, M_{i}$ and $M_{i+1}$, define $\Psi_{i}\left(s_{i}\right)=w_{i}, \Psi_{i}\left(s_{i+1}\right)=w_{i+1}, \Psi^{\prime \prime}\left(s_{i}\right)=M_{i}, \Psi^{\prime \prime}\left(s_{i+1}\right)=M_{i+1}$. Using the algebraic exercise, we get the following expression:

$$
A_{i}=s_{i}+\frac{M_{i}}{\tau^{2}}, \quad B_{i}=\frac{s_{i+1}-s_{i}}{h}+\frac{M_{i+1}-M_{i}}{\tau \theta}, C_{i}=\frac{M_{i} \cos \theta-M_{i+1}}{\tau^{2} \sin \theta}, L_{i}=-\frac{M_{i}}{\tau^{2}}, \quad \forall i=0,1,2, \ldots, N,
$$

where $\theta=\tau$. Hence $A_{i}, B_{i}, C_{i}$ and $L_{i}$ are depend on $\tau$ and $\theta$.

We obtain the subsequent relationships for $i=1,2, \ldots, \mathrm{N}-1$ by using the continuity of the first derivative, i.e. $\Psi_{i-1}^{\prime}\left(s_{i}\right)=\Psi_{i}^{\prime}\left(s_{i}\right)$, at $\left(s_{i}, w_{i}\right)$.

$$
\alpha M_{i+1}+2 \beta M_{i}+\alpha M_{i-1}=\frac{w_{i+1}-2 w_{i}+w_{i-1}}{h^{2}},
$$

where $\alpha=\frac{-1}{\theta^{2}}+\frac{1}{\theta \sin \theta}, \beta=\frac{1}{\theta^{2}}-\frac{\cos \theta}{\theta \sin \theta}, M_{j}=w^{\prime \prime}\left(s_{j}\right), j=i, i \pm 1$.

The local truncation error $T_{i}$ for $i=1,2, \ldots, N-1$, associated with the scheme Eq. (4) is

$$
T_{i}=h^{2}(1-2 \alpha-2 \beta) w_{i}^{(2)}+h^{4}\left(\frac{1-12 \alpha}{12}\right) w_{i}^{(4)}+h^{6}\left(\frac{1-30 \alpha}{360}\right) w_{i}^{(6)}+O\left(h^{8}\right) .
$$

Thus, for diverse values of $\alpha$ and $\beta$ in the scheme Eq. (4), specifies the different orders:

(i) for any choice of the arbitrary $\alpha$ and $\beta$ with $\alpha+\beta=\frac{1}{2}$, the truncation error is of fourth order.

(ii) for $\alpha=\frac{1}{12}, \quad \beta=\frac{5}{12}$, the truncation error is sixth order.

\section{Numerical scheme with small delay}

\subsection{Left - end boundary layer}

If the delay is of the small order of the perturbation parameter i.e., $\delta=o(\varepsilon)$, using Taylor's series on the term in Eq. (1) containing the delay parameter, it reduces to

$$
(\varepsilon-\delta P(s)) w^{\prime \prime}(s)+P(s) w^{\prime}(s)+Q(s) w(s)=R(s),
$$

subject to

$$
w(0)=\phi(0)=\phi_{0}, w(1)=\gamma .
$$

Assume that $P(s) \geq \bar{M}>0$ and $(\varepsilon-\delta P(s)) \geq 0$ throughout the domain [0,1], where $\bar{M}$ is a positive constant. With this hypothesis, for small values of $\varepsilon$, Eq. (5) show layer behaviour at $s=0$.

Let $\mathcal{L}^{\varepsilon}$ be the differential operator for the problem Eq. (5), Eq. (6) which is defined for any smooth function $\Omega(s) \in C^{(2)}$ as

$$
\mathcal{L}^{\varepsilon} \Omega(\mathrm{s})=\left(\varepsilon-\delta P_{i}\right) \Omega^{\prime \prime}(s)+P(s) \Omega^{\prime}(s)+Q(s) \Omega(s) .
$$


Case-(i). When $Q(s) \leq-\tau<0$, where $\tau$ is positive constant.

Lemma 4.1. Continuous minimum principle: Let $\Omega(s)$ is smooth function satisfying $\Omega_{0} \geq 0$ and $\Omega_{N} \geq 0$. Then $\mathcal{L}^{\varepsilon} \Omega(s) \leq 0, \forall i=1,2, \ldots, N-1$ implies that $\Omega(s) \geq 0, \forall i=$ $0,1,2, \ldots, N$.

Proof: Let $s^{*}$ be such that $\Omega\left(s^{*}\right)=\min _{0 \leq s \leq 1} \Omega(s)$ and assume that $\Omega\left(s^{*}\right)<0$. Clearly $s^{*} \notin\{0,1\}$, therefore $\Omega^{\prime}\left(s^{*}\right)=0$ and $\Omega^{\prime \prime}\left(s^{*}\right) \geq 0$.

Furthermore, $\mathcal{L}^{\varepsilon} \Omega\left(s^{*}\right)=(\varepsilon-\delta P) \Omega^{\prime \prime}\left(s^{*}\right)+P(s) \Omega^{\prime}\left(s^{*}\right)+Q(s) \Omega\left(s^{*}\right)>0$ contradicts the hypothesis that $\mathcal{L}^{\varepsilon} \Omega(s) \leq 0, \forall s \in(0,1)$, therefore our assumption that $\Omega\left(s^{*}\right)<0$ is wrong and thus $\Omega(s) \geq 0, \forall s \in\left[\begin{array}{ll}0 & 1\end{array}\right]$.

Lemma 4.2. Under the assumption that $P(s) \geq \bar{M}>0, Q(s) \leq-\tau<0$ where $\bar{M}, \tau$ are positive constants, the solution of Eq. (5) with boundary conditions Eq. (6) exists and satisfies

$$
\|w\| \leq \tau^{-1}\|R\|+\max \left(\varnothing_{0}, \gamma\right) \text {. }
$$

Proof: Construct the two barrier function $\pi^{ \pm}$defined by

$$
\pi^{ \pm}(s)=\tau^{-1}\|R\|+\max \left(\emptyset_{0}, \gamma\right) \pm \mathrm{w}(\mathrm{s}) .
$$

Then, we have

$$
\begin{aligned}
\pi^{ \pm}(0) & =\tau^{-1}\|R\|+\max \left(\emptyset_{0}, \gamma\right) \pm \mathrm{w}(0) \\
& =\tau^{-1}\|R\|+\max \left(\emptyset_{0}, \gamma\right) \pm \emptyset_{0} \\
& \geq 0 \\
\pi^{ \pm}(1) & =\tau^{-1}\|R\|+\max \left(\emptyset_{0}, \gamma\right) \pm \mathrm{w}(1) \\
& =\tau^{-1}\|R\|+\max \left(\emptyset_{0}, \gamma\right) \pm \gamma \\
& \geq 0,
\end{aligned}
$$

and

$$
\begin{aligned}
\mathcal{L}^{\varepsilon} \pi^{ \pm}(s) & =(\varepsilon-\delta P(s))\left(\pi^{ \pm}(s)\right)^{\prime \prime}+P(s)\left(\pi^{ \pm}(s)\right)^{\prime}+Q(s) \pi^{ \pm}(s) \\
& =Q(s)\left(\tau^{-1}\|R\|+\max \left(\emptyset_{0}, \gamma\right)\right) \pm \mathcal{L}^{\varepsilon} w(s) \\
& =Q(s)\left(\tau^{-1}\|R\|+\max \left(\emptyset_{0}, \gamma\right)\right) \pm R(s) .
\end{aligned}
$$

Since $Q(s) \leq-\tau<0$, i.e. $Q(s) \tau^{-1} \leq-1$ we get

$$
\mathcal{L}^{\varepsilon} \pi^{ \pm}(s)=(-\|R\| \pm R(\mathrm{~s}))+Q(s) \max \left(\emptyset_{0}, \gamma\right) \leq 0 .
$$

Therefore, using Lemma 4.1, we obtain $\pi^{ \pm}(s) \geq 0, \forall s \in[0,1]$, gives required estimate.

Case-(ii). When $Q(s) \geq \tau>0$, where $\tau$ is positive constant.

Lemma 4.3. Continuous maximum principle: Let $\Omega(s)$ is smooth function satisfying $\Omega_{0} \geq 0$ and $\Omega_{N} \geq 0$. Then $\mathcal{L}^{\varepsilon} \Omega(s) \geq 0, \forall i=1,2, \ldots, N-1$ implies that $\Omega(s) \geq 0, \forall i=$ $0,1,2, \ldots, N$.

Proof: Let $s^{*}$ be such that $\Omega\left(s^{*}\right)=\max _{0 \leq s \leq 1} \Omega(s)$ and assume that $\Omega\left(s^{*}\right)<0$. Clearly $s^{*} \notin$ $\{0,1\}$, therefore $\Omega^{\prime}\left(s^{*}\right)=0$ and $\Omega^{\prime \prime}\left(s^{*}\right) \leq 0$. Further $\mathcal{L}^{\varepsilon} \Omega\left(s^{*}\right)=(\varepsilon-\delta P) \Omega^{\prime \prime}\left(s^{*}\right)+$ 
$P(s) \Omega^{\prime}\left(s^{*}\right)+Q(s) \Omega\left(s^{*}\right) \leq 0$ which contradicts the hypothesis that $\mathcal{L}^{\varepsilon} \Omega(s) \geq 0, \forall s \in(0,1)$. Hence, our assumption that $\Omega\left(s^{*}\right)<0$ is wrong and thus $\Omega(s) \geq 0, \forall s \in\left[\begin{array}{ll}0 & 1\end{array}\right]$.

Lemma 4.4. Under the assumption that $P(s) \geq \bar{M}>0, Q(s) \geq \tau>0$ where $\bar{M}, \tau$ are positive constants, the solution of the Eq. (5) with boundary conditions Eq. (6) exists and satisfies

$$
\|w\| \leq \tau^{-1}\|R\|+\max \left(\emptyset_{0}, \gamma\right) .
$$

Proof: Let us construct the two barrier function $\pi^{ \pm}$defined by

$$
\pi^{ \pm}(s)=\tau^{-1}\|R\|+\max \left(\varnothing_{0}, \gamma\right) \pm \mathrm{w}(\mathrm{s}) .
$$

Then, we have

$$
\begin{aligned}
\pi^{ \pm}(0) & =\tau^{-1}\|R\|+\max \left(\emptyset_{0}, \gamma\right) \pm \mathrm{w}(0) \\
& =\tau^{-1}\|R\|+\max \left(\emptyset_{0}, \gamma\right) \pm \emptyset_{0} \\
& \geq 0, \\
\pi^{ \pm}(1) & =\tau^{-1}\|R\|+\max \left(\emptyset_{0}, \gamma\right) \pm \mathrm{w}(1) \\
& =\tau^{-1}\|R\|+\max \left(\emptyset_{0}, \gamma\right) \pm \gamma \\
& \geq 0,
\end{aligned}
$$

and

$$
\begin{aligned}
\mathcal{L}^{\varepsilon} \pi^{ \pm}(s) & =(\varepsilon-\delta P(s))\left(\pi^{ \pm}(s)\right)^{\prime \prime}+P(s)\left(\pi^{ \pm}(s)\right)^{\prime}+Q(s) \pi^{ \pm}(s) \\
& =Q(s)\left(\tau^{-1}\|R\|+\max \left(\emptyset_{0}, \gamma\right)\right) \pm \mathcal{L}^{\varepsilon} w(s) \\
& =Q(s)\left(\tau^{-1}\|R\|+\max \left(\emptyset_{0}, \gamma\right)\right) \pm R(s) .
\end{aligned}
$$

Since $Q(s) \geq \tau>0$, i.e. $Q(s) \tau^{-1} \geq 1$, we get

$$
\mathcal{L}^{\varepsilon} \pi^{ \pm}(s)=(\|R\| \pm \mathrm{R}(\mathrm{s}))+Q(s) \max \left(\emptyset_{0}, \gamma\right) \geq 0 .
$$

Therefore, using Lemma 4.3, we obtain $\pi^{ \pm}(s) \geq 0, \forall s \in[0,1]$, which gives required estimate.

Lemma 4.1 and 4.3 implies that the solution is unique and since the problem under the consideration is linear, the existence of the solution implied by its uniqueness. Additionally, Lemma 4.2 and 4.4 gives the boundedness of the solution (Ref. [12]).

Using the singular perturbation concept, the solution of Eq. (5) and Eq. (6) is of the structure (Ref. [21])

$$
w\left(s_{i}\right)=w_{0}\left(s_{i}\right)+\left(\phi(0)-w_{0}(0)\right) e^{-\left(\frac{P(0)}{\varepsilon-\delta P(0)}\right) s_{i}}+O(\varepsilon),
$$

i.e. $\quad w(i h)=w_{0}(i h)+\left(\phi(0)-w_{0}(0)\right) e^{-\left(\frac{P(0)}{\varepsilon-\delta P(0)}\right) i h}+O(\varepsilon)$.

Let $\rho=\frac{h}{\varepsilon-\delta p(0)}, \varepsilon-\delta p(0) \neq 0$, then Eq. (8) can be written as

$$
w(i h)=w_{0}(i h)+\left(\phi(0)-w_{0}(0)\right) e^{-(P(0)) i \rho}+O(\varepsilon) .
$$

Hence, $\quad \lim _{h \rightarrow 0} w(i h)=w_{0}(0)+\left(\phi(0)-w_{0}(0)\right) e^{-(P(0)) i \rho}+O(\varepsilon)$.

At the mesh point $s_{j}$, the Eq. (5) may be discretized by 


$$
\left(\varepsilon-\delta P\left(s_{j}\right)\right) M_{j}=R\left(s_{j}\right)-P\left(s_{j}\right) w_{j}^{\prime}(s)-Q\left(s_{j}\right) w\left(s_{j}\right), \forall j=i-1, i, i+1 .
$$

Now, use the following difference approximations to the first-order derivative of $w$ :

$$
w_{i-1}^{\prime}=\frac{-w_{i+1}+4 w_{i}-3 w_{i-1}}{2 h}+O\left(h^{2}\right), w_{i+1}^{\prime}=\frac{3 w_{i+1}-4 w_{i}+w_{i-1}}{2 h}+O\left(h^{2}\right), w_{i}^{\prime}=\frac{w_{i+1}-w_{i-1}}{2 h}+O\left(h^{2}\right) .
$$

Substituting the values of $M_{i-1}, M_{i}$ and $M_{i+1}$ along with the above differences in Eq. (10), we get

$$
\begin{gathered}
\frac{\left(\varepsilon-\delta P\left(s_{i}\right)\right)}{h^{2}}\left(w_{i+1}-2 w_{i}+w_{i-1}\right)=\left(\frac{-\alpha P_{i+1}}{2 h}+\frac{\beta P_{i}}{h}-\alpha Q_{i-1}+\frac{3 \alpha P_{i-1}}{2 h}\right) w_{i-1} \\
+\left(\frac{2 \alpha P_{i+1}}{h}-2 \beta Q_{i}-\frac{2 \alpha P_{i-1}}{h}\right) w_{i}+\left(\frac{-3 \alpha P_{i+1}}{2 h}-\frac{\beta P_{i}}{h}-\alpha Q_{i+1}+\frac{\alpha P_{i-1}}{2 h}\right) w_{i+1} \\
+\left(\alpha R_{i+1}+2 \beta R_{i}+\alpha R_{i-1}\right), \forall i=1,2, \ldots, N-1 .
\end{gathered}
$$

Introducing a fitting parameter $\sigma(\rho)$ in Eq. (11), we have

$$
\begin{gathered}
\frac{\sigma(\rho)\left(\varepsilon-\delta P\left(s_{i}\right)\right)}{h^{2}}\left(w_{i+1}-2 w_{i}+w_{i-1}\right)=\left(\frac{-\alpha P_{i+1}}{2 h}+\frac{\beta P_{i}}{h}-\alpha Q_{i-1}+\frac{3 \alpha P_{i-1}}{2 h}\right) w_{i-1} \\
+\left(\frac{2 \alpha P_{i+1}}{h}-2 \beta Q_{i}-\frac{2 \alpha P_{i-1}}{h}\right) w_{i}+\left(\frac{-3 \alpha P_{i+1}}{2 h}-\frac{\beta P_{i}}{h}-\alpha Q_{i+1}+\frac{\alpha P_{i-1}}{2 h}\right) w_{i+1} \\
+\left(\alpha R_{i+1}+2 \beta R_{i}+\alpha R_{i-1}\right), \quad \forall i=1,2, \ldots, N-1 .
\end{gathered}
$$

Using the procedure given in [4], we get

$$
\lim _{h \rightarrow 0} \frac{\sigma}{\rho}(w(i h+h)-2 w(i h)+w(i h-h))=\frac{P(0)}{2} \lim _{h \rightarrow 0}(w(i h-h)-w(i h+h)) .
$$

Using Eq. (9) into Eq. (13), we get the fitting parameter for the layer behaviour at the left-end point of the domain as:

$$
\sigma=\rho(\alpha+\beta) P(0) \operatorname{coth}\left(\frac{P(0) \rho}{2}\right)
$$

where $\rho=\frac{h}{\varepsilon-\delta P(0)}$.

Exercising on Eq. (12), we get the following tridiagonal system

$$
E_{i-1} w_{i-1}+F_{i} w_{i}+G_{i+1} w_{i+1}=H_{i}, \quad \forall i=1,2, \ldots, N-1,
$$

where $\quad E_{i-1}=-\sigma\left(\varepsilon-\delta P_{i}\right)+\frac{3 h \alpha P_{i-1}}{2}+\beta h P_{i}-\frac{\alpha h P_{i+1}}{2}-\alpha h^{2} Q_{i-1}$,

$$
F_{i}=2 \sigma\left(\varepsilon-\delta P_{i}\right)-2 \alpha h P_{i-1}+2 \alpha h P_{i+1}-2 \beta h^{2} Q_{i} \text {, }
$$




$$
\begin{gathered}
G_{i+1}=-\sigma\left(\varepsilon-\delta P_{i}\right)+\frac{\alpha h P_{i-1}}{2}-\beta h P_{i}-\frac{3 \alpha h P_{i+1}}{2}-\alpha h^{2} Q_{i+1}, \\
H_{i}=-h^{2}\left[\alpha R_{i-1}+2 \beta R_{i}+\alpha R_{i+1}\right], \\
P\left(s_{i}\right)=P_{i}, Q\left(s_{i}\right)=Q_{i}, R\left(s_{i}\right)=R_{i}, \forall i=1,2, \ldots, N-1 .
\end{gathered}
$$

The system Eq. (15) is resolved by applying the Thomas tridiagonal algorithm.

\subsection{Right - end boundary layer}

With the assumption $P(s) \leq \bar{M}<0$ and $(\varepsilon-\delta P(s)) \geq 0$ all over the domain $[0,1]$, where $\bar{M}$ is negative constant, for small values of $\varepsilon$, boundary layer for Eq. (5) exists at $s=1$. From the singular perturbation theory, the solution of Eq. (5) and Eq. (6) is of the form

i.e., $\quad w(i h)=w_{0}(i h)+\left(\phi(0)-w_{0}(1)\right) e^{-\left(\frac{P(1)}{\varepsilon-\delta P(1)}\right)(1-i h)}+O(\varepsilon)$,

$$
w(i h)=w_{0}(i h)+\left(\phi(0)-w_{0}(1)\right) e^{-P(1)\left(\frac{1}{\varepsilon-\delta P(1)}-i \rho\right)}+O(\varepsilon),
$$

where $\rho=\frac{h}{\varepsilon-\delta P(1)}, \varepsilon-\delta P(1) \neq 0$.

$$
\therefore \lim _{h \rightarrow 0} w(i h)=w_{0}(0)+\left(\phi(0)-w_{0}(1)\right) e^{-P(1)\left(\frac{1}{\varepsilon-\delta P(1)}-i \rho\right)}+O(\varepsilon) \text {. }
$$

Now, in the non-polynomial spline finite difference method Eq. (12), insert a fitting parameter $\sigma(\rho)$ and apply the same procedure as in left boundary layer case, we get the fitting parameter

$$
\sigma=\rho(\alpha+\beta) P(0) \operatorname{coth}\left(\frac{P(1) \rho}{2}\right) .
$$

In this case also, we have the tridiagonal system Eq. (15) where $\sigma$ is given by Eq. (19).

\section{Convergence analysis}

To examine the convergence for the scheme for the left-end layer, consider the matrix form of Eq. (15) including the specified boundary conditions [22]

$$
(\widehat{\mathcal{D}}+\widehat{\mathcal{P}}) W+\widehat{\mathcal{Q}}+\mathcal{T}(h)=0,
$$

where

$$
\widehat{\mathcal{D}}=\left[\begin{array}{cccccc}
2\left(\varepsilon-\delta P_{1}\right) \sigma & -\left(\varepsilon-\delta P_{1}\right) \sigma & 0 & 0 & . . & 0 \\
-\left(\varepsilon-\delta P_{2}\right) \sigma & 2\left(\varepsilon-\delta P_{2}\right) \sigma & -\left(\varepsilon-\delta P_{2}\right) \sigma & . . & . . & 0 \\
0 & . . & . . & . . & . . & 0 \\
. . & . . & . . & . . & . . & . . \\
. . & . . & . . & . . & . . & . . \\
0 & . . & . . & . . & -\left(\varepsilon-\delta P_{N-1}\right) \sigma & 2\left(\varepsilon-\delta P_{N-1}\right) \sigma
\end{array}\right],
$$




$$
\hat{\mathcal{P}}=\left[x_{i}, y_{i}, t_{i}\right]=\left[\begin{array}{cccccc}
y_{1} & t_{1} & 0 & 0 & \ldots \ldots \ldots & 0 \\
x_{2} & y_{2} & t_{2} & 0 & \ldots \ldots \ldots & 0 \\
0 & x_{3} & y_{3} & t_{3} & \ldots \ldots \ldots & 0 \\
. . & . . & . . & . . & . . & . . \\
. . & . . & . . & . . & . . & . . \\
0 & . . & . . & 0 & x_{N-1} & y_{N-1}
\end{array}\right],
$$

where

$$
\begin{aligned}
& x_{i}=\frac{3 h \alpha P_{i-1}}{2}+\beta h P_{i}-\frac{\alpha h P_{i+1}}{2}-\alpha h^{2} Q_{i-1}, \quad y_{i}=-2 \alpha h P_{i-1}+2 \alpha h P_{i+1}-2 \beta h^{2} Q_{i}, \\
& t_{i}=\frac{\alpha h P_{i-1}}{2}-\beta h P_{i}-\frac{3 \alpha h P_{i+1}}{2}-\alpha h^{2} Q_{i+1}, \forall i=1,2, \ldots, N-1, \text { and } \\
& \hat{\mathcal{Q}}=\left[v_{1}+\left(-\left(\varepsilon-\delta P_{1}\right) \sigma+x_{1}\right) \phi_{0}, v_{2}, v_{3}, \ldots, v_{N-2}, v_{N-1}+\left(-\left(\varepsilon-\delta P_{N-1}\right) \sigma+t_{N-1}\right) \gamma\right]^{T},
\end{aligned}
$$$$
\text { where } v_{i}=h^{2}\left[\alpha R_{i-1}+2 \beta R_{i}+\alpha R_{i+1}\right] \quad i=1,2, \ldots, N-1, T(h)=O\left(h^{4}\right) \text { and }
$$
$W=\left[W_{1}, W_{2}, \ldots, W_{N-1}\right]^{T}, \mathcal{T}(h)=\left[\mathcal{T}_{1}, \mathcal{T}_{2}, \ldots, \mathcal{T}_{N-1}\right]^{T}, O=[0,0, \ldots, 0]^{T} \quad$ are $\quad$ associated vectors of Eq. (20).

Let $w=\left[w_{1}, w_{2}, \ldots, w_{N-1}\right]^{T} \cong W$ which satisfies the equation

$$
(\widehat{\mathcal{D}}+\widehat{\mathcal{P}}) w+\hat{\mathcal{Q}}=0 .
$$

Let $e_{i}=w_{i}-W_{i}, \forall i=1,2 \ldots, N-1$ be the discretization error so that $E=\left[e_{1}, e_{2}, \ldots, e_{N-1}\right]^{T}=w-W$. Subtracting Eq. (20) from Eq. (21), we get the error equation

$$
(\widehat{\mathcal{D}}+\widehat{\mathcal{P}}) E=\mathcal{T}(h)
$$

Let $|P(s)| \leq \tilde{C}_{1}$ and $|Q(s)| \leq \tilde{C}_{2}$ where $\tilde{C}_{1}, \tilde{C}_{2}$ are positive constants. If $\zeta_{i, j}$ be the $(i, j)^{t h}$ element of the matrix $(\bar{D}+\bar{P})$, then

$$
\begin{gathered}
\left|\zeta_{i, i+1}\right| \leq\left(\varepsilon-\delta P_{i}\right)+h(\alpha+\beta) \tilde{C}_{1}+h^{2} \alpha \tilde{C}_{2}, \forall i=1,2, \ldots, N-2, \\
\left|\zeta_{i, i-1}\right| \leq\left(\varepsilon-\delta P_{i}\right)+h(\alpha+\beta) \tilde{C}_{1}+h^{2} \alpha \tilde{C}_{2}, \forall i=2,3, \ldots, N-1 .
\end{gathered}
$$

Thus, for small values of $h$, we have

$$
\begin{gathered}
\left|\zeta_{i, i+1}\right|<\left(\varepsilon-\delta P_{i}\right), \quad \forall i=1,2, \ldots, N-2, \\
\left|\zeta_{i, i-1}\right|<\left(\varepsilon-\delta P_{i}\right), \quad \forall i=2,3, \ldots, N-1 .
\end{gathered}
$$

Hence $(\widehat{\mathcal{D}}+\widehat{\mathcal{P}})$ is irreducible [27].

Let the sum of $i^{\text {th }}$ row elements of the matrix $(\widehat{\mathcal{D}}+\widehat{\mathcal{P}})$ be $\mathbb{S}_{i}$, then we have

$$
\begin{aligned}
& \mathbb{S}_{i}=\left(\varepsilon-\delta P_{i}\right) \sigma-\frac{3 \alpha h P_{i-1}}{2}-\beta h P_{i}+\frac{\alpha h P_{i+1}}{2}-h^{2}\left(2 \beta Q_{i}+\alpha Q_{i+1}\right), i=1, \\
& \mathbb{S}_{i}=h^{2}\left(\alpha Q_{\mathrm{i}-1}+2 \beta Q_{i}+\alpha Q_{i+1}\right), \forall i=2,3, \ldots, N-2, \\
& \mathbb{S}_{i}=\left(\varepsilon-\delta P_{i}\right) \sigma-\frac{\alpha h P_{i-1}}{2}-\beta h P_{i}+\frac{3 \alpha h P_{i+1}}{2}-h^{2}\left(2 \beta Q_{i}+\alpha Q_{i-1}\right), i=N-1 .
\end{aligned}
$$

Let $\tilde{C}_{1^{*}}=\min _{1 \leq i \leq N}|P(s)|$ and $\tilde{C}_{1}^{*}=\max _{1 \leq i \leq N}|P(s)|, \tilde{C}_{2^{*}}=\min _{1 \leq i \leq N}|Q(s)|$ and $\tilde{C}_{2}^{*}=\max _{1 \leq i \leq N}|Q(s)|$. 
Since $0<\varepsilon \ll 1$ and $\varepsilon \propto O(h)$, it is verified that for sufficiently small $h,(\widehat{\mathcal{D}}+\widehat{\mathcal{P}})$ is monotone $[27,28]$. Hence $(\widehat{\mathcal{D}}+\widehat{\mathcal{P}})^{-1}$ exists and $(\widehat{\mathcal{D}}+\widehat{\mathcal{P}})^{-1} \geq 0$. Thus from Eq. (22), we have

$$
\|E\| \leq\left\|(\widehat{\mathcal{D}}+\widehat{\mathcal{P}})^{-1}\right\|\|\mathcal{T}\| .
$$

Let $(\widehat{\mathcal{D}}+\widehat{\mathcal{P}})_{i, k}^{-1}$ be the $(i, k)^{\text {th }}$ element of $(\widehat{\mathcal{D}}+\widehat{\mathcal{P}})^{-1}$ and define

$$
\left\|(\widehat{\mathcal{D}}+\widehat{\mathcal{P}})^{-1}\right\|=\max _{1 \leq i \leq N-1} \sum_{k=1}^{N-1}(\widehat{\mathcal{D}}+\widehat{\mathcal{P}})_{i, k}^{-1} \text { and }\|\mathcal{T}(h)\|=\max _{1 \leq i \leq N-1}|\mathcal{T}(h)| .
$$

Since $(\widehat{\mathcal{D}}+\widehat{\mathcal{P}})_{i, k}^{-1} \geq 0$ and $\sum_{k=1}^{N-1}(\widehat{\mathcal{D}}+\widehat{\mathcal{P}})_{i, k}^{-1} . \mathbb{S}_{k}=1, \forall i=1,2, \ldots, N-1$.

Hence,

$$
\begin{gathered}
(\widehat{\mathcal{D}}+\widehat{\mathcal{P}})_{i, k}^{-1} \leq \frac{1}{\mathbb{S}_{i}}<\frac{1}{h^{2} \tilde{C}_{2}}, \quad i=1, \\
(\widehat{\mathcal{D}}+\widehat{\mathcal{P}})_{i, k}^{-1} \leq \frac{1}{\mathbb{S}_{i}}<\frac{1}{h^{2} \tilde{C}_{2}}, \quad i=N-1 .
\end{gathered}
$$

Furthermore,

$$
\sum_{k=1}^{N-1}(\widehat{\mathcal{D}}+\widehat{\mathcal{P}})_{i, k}^{-1} \leq \frac{1}{\min _{2 \leq i \leq N-2} \mathbb{S}_{i}}<\frac{1}{h^{2} \tilde{C}_{2}}, \forall i=1,2, \ldots, N-1 .
$$

By the help of Eqs. (25a) - (25d) and using Eq. (24) we get

$$
\|E\| \leq O\left(h^{2}\right) .
$$

Hence, the proposed scheme has second-order convergence.

\section{Numerical examples}

To illustrate the comparative efficiency of the proposed scheme, it is implemented on numerical experiments with a layer at left-end and right-end for the small $\delta$ values. The maximum absolute errors in the examples considered were measured using the theory of double mesh $E^{N}=\max _{0 \leq i \leq N}\left|w_{i}^{N}-w_{2 i}^{2 N}\right|$ and tabulated in Tables $1-5$ for the examples considered. Computed errors are compared to the results given in $[12,23,24]$. It was observed that our method yields precise results than the method suggested in [12, 23, 24].

Example 6.1. $\varepsilon w^{\prime \prime}(s)+w^{\prime}(s-\delta)-w(s)=0$ with $w(s)=1,-\delta \leq s \leq 0 ; w(1)=1$.

Example 6.2. $\varepsilon w^{\prime \prime}(s)+(1+s) w^{\prime}(s-\delta)-e^{-s} w(t)=1 ; w(s)=0,-\delta \leq s \leq 0$; $w(1)=1$.

Example 6.3. $\varepsilon w^{\prime \prime}(s)-w^{\prime}(s-\delta)-w(s)=0 ; w(s)=1,-\delta \leq s \leq 0 ; w(1)=-1$.

Example 6.4. $\varepsilon w^{\prime \prime}(s)-e^{s} w^{\prime}(s-\delta)-s w(s)=0, w(s)=1,-\delta \leq s \leq 0 ; w(1)=1$. 
Example 6.5. Consider the following singularly perturbed nonlinear delay differential equation

$$
\varepsilon w^{\prime \prime}(s)+w(s) w^{\prime}(s-\delta)-w(s)=0,
$$

under the interval and boundary conditions $w(s)=1,-\delta \leq s \leq 0, w(1)=1$.

Table 1. The maximum absolute errors in Example 6.1 with $\varepsilon=0.1$

\begin{tabular}{|l|l|l|l|l|l|l|}
\hline \multirow{2}{*}{$N$} & \multicolumn{2}{|c|}{$\delta=0.3 \times \varepsilon$} & \multicolumn{2}{c|}{$\delta=0.6 \times \varepsilon$} & \multicolumn{2}{c|}{$\delta=0.9 \times \varepsilon$} \\
\cline { 2 - 7 } & Our method & $\begin{array}{c}\text { Results in } \\
{[12]}\end{array}$ & Our method & $\begin{array}{c}\text { Results in } \\
{[12]}\end{array}$ & Our method & $\begin{array}{c}\text { Results in } \\
{[12]}\end{array}$ \\
\hline 64 & $9.30 \mathrm{e}-04$ & $3.42 \mathrm{e}-03$ & $1.40 \mathrm{e}-03$ & $3.43 \mathrm{e}-03$ & $1.40 \mathrm{e}-03$ & $3.43 \mathrm{e}-03$ \\
128 & $2.66 \mathrm{e}-04$ & $1.66 \mathrm{e}-03$ & $5.97 \mathrm{e}-04$ & $1.66 \mathrm{e}-03$ & $7.13 \mathrm{e}-04$ & $1.66 \mathrm{e}-03$ \\
256 & $6.93 \mathrm{e}-05$ & $8.11 \mathrm{e}-04$ & $1.94 \mathrm{e}-04$ & $8.15 \mathrm{e}-04$ & $3.58 \mathrm{e}-04$ & $8.12 \mathrm{e}-04$ \\
512 & $1.75 \mathrm{e}-05$ & $4.03 \mathrm{e}-04$ & $5.27 \mathrm{e}-05$ & $4.02 \mathrm{e}-04$ & $1.79 \mathrm{e}-04$ & $4.03 \mathrm{e}-04$ \\
\hline
\end{tabular}

Table 2. The maximum absolute errors in Example 6.2 with $\varepsilon=0.1$

\begin{tabular}{|l|l|l|l|l|}
\hline \multirow{2}{*}{$N$} & \multicolumn{2}{|c|}{$\delta=0.1 \times \varepsilon$} & \multicolumn{2}{c|}{$\delta=0.4 \times \varepsilon$} \\
\cline { 2 - 5 } & Our method & $\begin{array}{c}\text { Results in } \\
{[12]}\end{array}$ & Our method & $\begin{array}{c}\text { Results in } \\
{[12]}\end{array}$ \\
\hline 64 & $4.18 \mathrm{e}-04$ & $8.10 \mathrm{e}-03$ & $5.19 \mathrm{e}-04$ & $1.00 \mathrm{e}-02$ \\
128 & $1.74 \mathrm{e}-04$ & $5.11 \mathrm{e}-03$ & $2.58 \mathrm{e}-04$ & $5.07 \mathrm{e}-03$ \\
256 & $1.21 \mathrm{e}-04$ & $2.55 \mathrm{e}-03$ & $2.21 \mathrm{e}-04$ & $2.57 \mathrm{e}-03$ \\
512 & $1.15 \mathrm{e}-04$ & $1.29 \mathrm{e}-03$ & $2.33 \mathrm{e}-04$ & $1.26 \mathrm{e}-03$ \\
\hline
\end{tabular}

Table 3. The maximum absolute errors in Example 6.3 with $\varepsilon=0.01$

\begin{tabular}{|l|c|c|c|c|c|c|}
\hline \multirow{3}{*}{$\delta$} & \multicolumn{2}{|c|}{$N=10^{2}$} & \multicolumn{2}{c|}{$N=10^{3}$} & \multicolumn{2}{c|}{$N=10^{4}$} \\
\cline { 2 - 7 } & $\begin{array}{c}\text { Our } \\
\text { method }\end{array}$ & $\begin{array}{c}\text { Results in } \\
{[24]}\end{array}$ & $\begin{array}{c}\text { Our } \\
\text { method }\end{array}$ & $\begin{array}{c}\text { Results in } \\
{[24]}\end{array}$ & $\begin{array}{c}\text { Our } \\
\text { method }\end{array}$ & $\begin{array}{c}\text { Results in } \\
{[24]}\end{array}$ \\
\hline 0.000 & $2.00 \mathrm{e}-03$ & $1.81 \mathrm{e}-01$ & $2.03 \mathrm{e}-04$ & $2.42 \mathrm{e}-02$ & $4.90 \mathrm{e}-06$ & $2.51 \mathrm{e}-03$ \\
0.007 & $2.00 \mathrm{e}-03$ & $1.20 \mathrm{e}-01$ & $1.55 \mathrm{e}-04$ & $1.45 \mathrm{e}-02$ & $1.63 \mathrm{e}-06$ & $1.48 \mathrm{e}-03$ \\
0.015 & $2.00 \mathrm{e}-03$ & $8.66 \mathrm{e}-02$ & $8.48 \mathrm{e}-05$ & $9.96 \mathrm{e}-03$ & $7.52 \mathrm{e}-07$ & $1.01 \mathrm{e}-03$ \\
0.025 & $2.00 \mathrm{e}-03$ & $6.46 \mathrm{e}-02$ & $3.86 \mathrm{e}-05$ & $7.17 \mathrm{e}-03$ & $3.82 \mathrm{e}-07$ & $7.20 \mathrm{e}-04$ \\
\hline
\end{tabular}


Table 4. The maximum absolute errors in Example 6.4 with $\varepsilon=0.1$

\begin{tabular}{|c|l|c|c|c|c|c|}
\hline \multirow{2}{*}{$\delta$} & \multicolumn{2}{|c|}{$N=10^{2}$} & \multicolumn{2}{c|}{$N=10^{3}$} & \multicolumn{2}{c|}{$N=10^{4}$} \\
\cline { 2 - 7 } & Our method & $\begin{array}{c}\text { Results in } \\
{[24]}\end{array}$ & Our method & $\begin{array}{c}\text { Results in } \\
{[24]}\end{array}$ & $\begin{array}{c}\text { Our } \\
\text { method }\end{array}$ & $\begin{array}{c}\text { Results in } \\
{[24]}\end{array}$ \\
\hline 0.01 & $5.27 \mathrm{e}-04$ & $5.75 \mathrm{e}-03$ & $5.73 \mathrm{e}-06$ & $5.08 \mathrm{e}-04$ & $5.74 \mathrm{e}-08$ & $5.02 \mathrm{e}-05$ \\
0.03 & $2.65 \mathrm{e}-04$ & $3.93 \mathrm{e}-03$ & $2.73 \mathrm{e}-06$ & $3.61 \mathrm{e}-04$ & $2.74 \mathrm{e}-08$ & $3.58 \mathrm{e}-05$ \\
0.06 & $1.23 \mathrm{e}-04$ & $2.70 \mathrm{e}-03$ & $1.24 \mathrm{e}-06$ & $2.55 \mathrm{e}-04$ & $1.24 \mathrm{e}-08$ & $2.53 \mathrm{e}-05$ \\
0.08 & $8.19 \mathrm{e}-05$ & $2.24 \mathrm{e}-03$ & $8.23 \mathrm{e}-07$ & $2.14 \mathrm{e}-04$ & $8.22 \mathrm{e}-09$ & $2.13 \mathrm{e}-05$ \\
\hline
\end{tabular}

Table 5. Maximum errors in Example 6.5 for $\delta=0.8 \varepsilon$ and different values of $\varepsilon$ and $N$

\begin{tabular}{lccllll}
\hline$\varepsilon / N$ & 64 & 128 & 256 & 512 & 1024 & 2048 \\
\hline \multicolumn{2}{l}{ Our method } & & & & & \\
$10^{-1}$ & $1.1651 \mathrm{e}-03$ & $3.2884 \mathrm{e}-04$ & $8.7186 \mathrm{e}-05$ & $2.2435 \mathrm{e}-05$ & $5.6893 \mathrm{e}-06$ & $1.4325 \mathrm{e}-06$ \\
$10^{-2}$ & $3.3515 \mathrm{e}-03$ & $1.0682 \mathrm{e}-03$ & $2.9959 \mathrm{e}-04$ & $7.9176 \mathrm{e}-05$ & $2.0341 \mathrm{e}-05$ & $5.1542 \mathrm{e}-06$ \\
$10^{-3}$ & $3.8819 \mathrm{e}-03$ & $1.2730 \mathrm{e}-03$ & $3.6165 \mathrm{e}-04$ & $9.6155 \mathrm{e}-05$ & $2.4775 \mathrm{e}-05$ & $6.2866 \mathrm{e}-06$ \\
$10^{-4}$ & $3.9415 \mathrm{e}-03$ & $1.2967 \mathrm{e}-03$ & $3.6893 \mathrm{e}-04$ & $9.8159 \mathrm{e}-05$ & $2.5299 \mathrm{e}-05$ & $6.4208 \mathrm{e}-06$ \\
$10^{-5}$ & $3.9476 \mathrm{e}-03$ & $1.2991 \mathrm{e}-03$ & $3.6967 \mathrm{e}-04$ & $9.8363 \mathrm{e}-05$ & $2.5352 \mathrm{e}-05$ & $6.4344 \mathrm{e}-06$ \\
$10^{-6}$ & $3.9482 \mathrm{e}-03$ & $1.2994 \mathrm{e}-03$ & $3.6974 \mathrm{e}-04$ & $9.8383 \mathrm{e}-05$ & $2.5358 \mathrm{e}-05$ & $6.4358 \mathrm{e}-06$ \\
$10^{-7}$ & $3.9482 \mathrm{e}-03$ & $1.2994 \mathrm{e}-03$ & $3.6975 \mathrm{e}-04$ & $9.8385 \mathrm{e}-05$ & $2.5358 \mathrm{e}-05$ & $6.4359 \mathrm{e}-06$ \\
$10^{-8}$ & $3.9482 \mathrm{e}-03$ & $1.2994 \mathrm{e}-03$ & $3.6975 \mathrm{e}-04$ & $9.8385 \mathrm{e}-05$ & $2.5358 \mathrm{e}-05$ & $6.4359 \mathrm{e}-06$ \\
& & & & & & \\
Results $\mathrm{in} \mathrm{[23]}$ & & & & & \\
$10^{-1}$ & $6.036 \mathrm{E}-02$ & $1.973 \mathrm{E}-02$ & $5.134 \mathrm{E}-03$ & $1.276 \mathrm{E}-03$ & $3.185 \mathrm{E}-04$ & $7.963 \mathrm{E}-05$ \\
$10^{-2}$ & $5.894 \mathrm{E}-02$ & $1.902 \mathrm{E}-02$ & $5.945 \mathrm{E}-03$ & $1.812 \mathrm{E}-03$ & $5.279 \mathrm{E}-04$ & $1.444 \mathrm{E}-04$ \\
$10^{-3}$ & $5.891 \mathrm{E}-02$ & $1.895 \mathrm{E}-02$ & $5.906 \mathrm{E}-03$ & $1.791 \mathrm{E}-03$ & $5.164 \mathrm{E}-04$ & $1.382 \mathrm{E}-04$ \\
$10^{-4}$ & $5.893 \mathrm{E}-02$ & $1.902 \mathrm{E}-02$ & $5.921 \mathrm{E}-03$ & $1.789 \mathrm{E}-03$ & $5.153 \mathrm{E}-04$ & $1.376 \mathrm{E}-04$ \\
$10^{-5}$ & $5.892 \mathrm{E}-02$ & $1.901 \mathrm{E}-02$ & $5.943 \mathrm{E}-03$ & $1.806 \mathrm{E}-03$ & $5.172 \mathrm{E}-04$ & $1.376 \mathrm{E}-04$ \\
$10^{-6}$ & $5.899 \mathrm{E}-02$ & $1.901 \mathrm{E}-02$ & $5.938 \mathrm{E}-03$ & $1.806 \mathrm{E}-03$ & $5.250 \mathrm{E}-04$ & $1.409 \mathrm{E}-04$ \\
$10^{-7}$ & $5.973 \mathrm{E}-02$ & $1.902 \mathrm{E}-02$ & $5.938 \mathrm{E}-03$ & $1.805 \mathrm{E}-03$ & $5.237 \mathrm{E}-04$ & $1.420 \mathrm{E}-04$ \\
$10^{-8}$ & $6.713 \mathrm{E}-02$ & $1.915 \mathrm{E}-02$ & $5.940 \mathrm{E}-03$ & $1.805 \mathrm{E}-03$ & $5.234 \mathrm{E}-04$ & $1.416 \mathrm{E}-04$ \\
& & & & & & \\
\hline
\end{tabular}

\section{Numerical scheme without fitting factor for big delay}

If the delay $\delta=o(\varepsilon)$, the layer behaviour of the solution is preserved in both the cases i.e. Taylor's series for the term holding the shift parameter and the special mesh-type developed in [11]. If the delay parameter $\delta(\varepsilon)$ is of order $O(\varepsilon)$, the layer behaviour of the solution is no longer preserved and oscillatory behaviour is shown in the solution. 
For this reason, with the help of a special type mesh developed in [11], we are building a numerical scheme consisting of a non-polynomial spline method i.e. the term shift parameter is lying on the mesh point after discretization and selects the mesh as $h=\frac{\delta}{m}$, where $m=k l, l$ is the mantissa of $\delta$ and $k$ is a positive integer.

Using this mesh, Eq. (1) and (2) leads to

$$
\varepsilon w_{i}^{\prime \prime}=r\left(s_{i}\right)-P\left(s_{i}\right) w_{i-m}^{\prime}-Q\left(s_{i}\right) w_{i},
$$

with

$$
w_{i}=\phi_{i}, \forall i=-m,-m+1, \ldots, 0 \text { and } w_{N}=\gamma \text {. }
$$

Now use the Eq. (4) from the non-polynomial spline together with the following finite difference approximations of the first-order derivatives,

$w_{i-1}^{\prime}=\frac{-w_{i+1}+4 w_{i}-3 w_{i-1}}{2 h}+O\left(h^{2}\right), w_{i+1}^{\prime}=\frac{3 w_{i+1}-4 w_{i}+w_{i-1}}{2 h}+O\left(h^{2}\right), w_{i}^{\prime}=\frac{w_{i+1}-w_{i-1}}{2 h}+O\left(h^{2}\right)$, we get

$$
\begin{aligned}
\frac{\varepsilon}{h^{2}}\left(w_{i+1}-2 w_{i}\right. & \left.+w_{i-1}\right) \\
& =\left(\frac{-\alpha P_{i+1}}{2 h}+\frac{\beta P_{i}}{h}+\frac{3 \alpha P_{i-1}}{2 h}\right) w_{i-m-1}+\left(\frac{2 \alpha P_{i+1}}{h}-\frac{2 \alpha P_{i-1}}{h}\right) w_{i-m} \\
& +\left(\frac{-3 \alpha P_{i+1}}{2 h}-\frac{\beta P_{i}}{h}+\frac{\alpha P_{i-1}}{2 h}\right) w_{i-m+1}-\alpha Q_{i-1} w_{i-1}-2 \beta Q_{i} w_{i}-\alpha Q_{i+1} w_{i+1} \\
& +\left(\alpha R_{i+1}+2 \beta R_{i}+\alpha R_{i-1}\right) .
\end{aligned}
$$

Exercising on Eq. (29), the difference scheme becomes

$$
E_{i-1} w_{i-1}+F_{i} w_{i}+G_{i+1} w_{i+1}+U_{i} w_{i-m+1}+V_{i} w_{i-m}+\widetilde{W}_{i} w_{i-m-1}=H_{i},
$$

$\forall i=1,2, \ldots, N-1$. Here

$$
\begin{aligned}
E_{i-1} & =\frac{\varepsilon}{h^{2}}+\alpha Q_{i-1}, F_{i}=-\frac{2 \varepsilon}{h^{2}}+2 \beta Q_{i}, G_{i+1}=\frac{\varepsilon}{h^{2}}+\alpha Q_{i+1}, U_{i}=-\frac{\alpha P_{i-1}}{2 h}+\frac{2 \beta P_{i}}{2 h}+\frac{3 \alpha P_{i+1}}{2 h}, \\
V_{i} & =\frac{2 \alpha P_{i-1}}{h}-\frac{2 \alpha P_{i+1}}{h}, \widetilde{W}_{i}=-\frac{3 \alpha P_{i-1}}{2 h}-\frac{2 \beta P_{i}}{2 h}+\frac{\alpha P_{i+1}}{2 h}, H_{i}=\alpha R_{i-1}+2 \beta R_{i}+\alpha R_{i+1} .
\end{aligned}
$$

By using the boundary conditions Eq. (28), the scheme Eq. (30) can be written as

$$
\begin{aligned}
& E_{i-1} w_{i-1}+F_{i} w_{i}+G_{i+1} w_{i+1}=H_{i}-U_{i} w_{i-m+1}-V_{i} w_{i-m}-\widetilde{W}_{i} w_{i-m-1}, \forall 1 \leq i \leq m-1, \\
& E_{i-1} w_{i-1}+F_{i} w_{i}+G_{i+1} w_{i+1}+U_{i} w_{i-m+1}=H_{i}-V_{i} w_{i-m}-\widetilde{W}_{i} w_{i-m-1} \text { for } i=m, \\
& E_{i-1} w_{i-1}+F_{i} w_{i}+G_{i+1} w_{i+1}+U_{i} w_{i-m+1}+V_{i} w_{i-m}=H_{i}-\widetilde{W}_{i} w_{i-m-1} \text { for } i=m+1, \\
& E_{i-1} w_{i-1}+F_{i} w_{i}+G_{i+1} w_{i+1}+U_{i} w_{i-m+1}+V_{i} w_{i-m}+\widetilde{W}_{i} w_{i-m-1}=H_{i}, \forall m+2 \leq i \leq N-1 .
\end{aligned}
$$


The system of Eq. (31) is solved using partial pivoting in the Gauss elimination method.

Using the expansion of Taylor's series about $w_{i-m}$ on Eq. (30) and with Eq. (27), we get the local truncation error to the scheme as

$$
\begin{aligned}
& T_{i}(h)=\varepsilon[1-2(\alpha+\beta)] h^{2} w_{i}^{(2)}\left(\xi_{i}\right) \\
& +\left\{\frac{\varepsilon}{12}[1-12 \alpha] w_{i}^{(4)}\left(\xi_{i}\right)+\left[\frac{1}{3}[-2 \alpha+\beta] p_{i}\left(\xi_{i}\right) w_{i}^{(3)}\left(\xi_{i}\right)\right]\right\} h^{4}+O\left(h^{6}\right), \forall s_{i} \leq \xi_{i} \leq s_{i+1} .
\end{aligned}
$$

It is very clear that $T_{i}(h)=O\left(h^{4}\right)$ for any arbitrary choice of $\alpha$ and $\beta$ whose sum is equal to $\frac{1}{2}$. Thus, the above scheme is a second-order convergence.

\section{Numerical scheme with a fitting parameter for big delay}

The behaviour of the layer can modify its character and even get damaged if the delay is greater than that of a perturbation parameter, or oscillating behaviour can occur even in the case of a given type mesh [11]. In this respect, we have tried to introduce a fitting parameter into the non-polynomial spline scheme, which was defined in the previous section, with the special type mesh. The fitting parameter is determined by the theory of singular perturbations. Now, inserting a fitting parameter $\sigma(\rho)$ in Eq. (29), we get

$$
\begin{aligned}
\frac{\sigma(\rho) \varepsilon}{h^{2}}\left(w_{i+1}\right. & \left.-2 w_{i}+w_{i-1}\right) \\
& =\left(\frac{-\alpha P_{i+1}}{2 h}+\frac{\beta P_{i}}{h}+\frac{3 \alpha P_{i-1}}{2 h}\right) w_{i-m-1} \\
& +\left(\frac{2 \alpha P_{i+1}}{h}-\frac{2 \alpha P_{i-1}}{h}\right) w_{i-m}+\left(\frac{-3 \alpha P_{i+1}}{2 h}-\frac{\beta P_{i}}{h}+\frac{\alpha P_{i-1}}{2 h}\right) w_{i-m+1} \\
& -\alpha Q_{i-1} w_{i-1}-2 \beta Q_{i} w_{i}-\alpha Q_{i+1} w_{i+1}+\left(\alpha R_{i+1}+2 \beta R_{i}+\alpha R_{i-1}\right),
\end{aligned}
$$

$\forall i=1,2, \ldots, N-1$.

Multiplying Eq. (32) by $h$ and taking the limit as $h \rightarrow 0$, we get

$$
\begin{aligned}
\lim _{h \rightarrow 0} \frac{\sigma}{\rho}(w(i h+ & h)-2 w(i h)+w(i h-h)) \\
= & (\alpha+\beta) p(0) \lim _{h \rightarrow 0}(w(i h-m h-h)-w(i h-m h+h)) .
\end{aligned}
$$

Using Eq. (9) into Eq. (33) and exercising, we get

$$
\sigma=\rho(\alpha+\beta) P(0) e^{P(0) m \rho} \operatorname{coth}\left(\frac{P(0) \rho}{2}\right),
$$

is the fitting parameter for the layer at left-end. 
Using the procedure as in right-end layer on Eq. (32) and exercising, we get

$$
\sigma=\rho(\alpha+\beta) P(0) e^{-P(0) m \rho} \operatorname{coth}\left(\frac{P(1) \rho}{2}\right),
$$

is the fitting parameter for the layer at right-end. Hence, the difference scheme for Eq. (32) is

$E_{i-1} w_{i-1}+F_{i} w_{i}+G_{i+1} w_{i+1}+U_{i} w_{i-m+1}+V_{i} w_{i-m}+\widetilde{W}_{i} w_{i-m-1}=H_{i}, \forall i=1,2, \ldots, N-1$.

Here, $E_{i-1}=\frac{\sigma \varepsilon}{h^{2}}+\alpha Q_{i-1}, \quad F_{i}=-\frac{2 \sigma \varepsilon}{h^{2}}+2 \beta Q_{i}, \quad G_{i+1}=\frac{\varepsilon \sigma}{h^{2}}+\alpha Q_{i+1}, U_{i}=-\frac{\alpha P_{i-1}}{2 h}+\frac{2 \beta P_{i}}{2 h}+$ $\frac{3 \alpha P_{i+1}}{2 h}, V_{i}=\frac{2 \alpha P_{i-1}}{h}-\frac{2 \alpha P_{i+1}}{h}, \widetilde{W}_{i}=-\frac{3 \alpha P_{i-1}}{2 h}-\frac{2 \beta P_{i}}{2 h}+\frac{\alpha P_{i+1}}{2 h}, G_{i}=\alpha R_{i-1}+2 \beta R_{i}+\alpha R_{i+1}$.

Utilizing the boundary conditions Eq. (28), the scheme Eq. (36) can be written as

$$
\begin{aligned}
& E_{i-1} w_{i-1}+F_{i} w_{i}+G_{i+1} w_{i+1}=H_{i}-U_{i} w_{i-m+1}-V_{i} w_{i-m}-\widetilde{W}_{i} w_{i-m-1}, \forall 1 \leq i \leq m-1, \\
& E_{i-1} w_{i-1}+F_{i} w_{i}+G_{i+1} w_{i+1}+U_{i} w_{i-m+1}=H_{i}-V_{i} w_{i-m}-\widetilde{W}_{i} w_{i-m-1} \text { for } i=m, \\
& E_{i-1} w_{i-1}+F_{i} w_{i}+G_{i+1} w_{i+1}+U_{i} w_{i-m+1}+V_{i} w_{i-m}=H_{i}-\widetilde{W}_{i} w_{i-m-1} \quad \text { for } i=m+1, \\
& E_{i-1} w_{i-1}+F_{i} w_{i}+G_{i+1} w_{i+1}+U_{i} w_{i-m+1}+V_{i} w_{i-m}+\widetilde{W}_{i} w_{i-m-1}=H_{i}, \forall m+2 \leq i \leq N-1 .
\end{aligned}
$$

The system Eq. (37) is solved by partial pivoting in the Gauss elimination method.

\section{Numerical illustrations}

To demonstrate the method competence, three problems are discussed. Using the principle of double mesh $E^{N}=\max _{0 \leq i \leq N}\left|w_{i}^{N}-w_{2 i}^{2 N}\right|$, with and without fitting parameter, the maximum errors for the examples are calculated. The fitting parameter introduced in the scheme Eq. (32) is to handle the layer behaviour, when the shift is larger than the perturbation. The computed solutions of the problem are illustrated through the graphs, with and without fitting parameter for different values of and of $\delta$. Tables 6-11 display for the maximum absolute errors for the examples. The results in [11] are compared to the computed errors. It was found that the method proposed provides solutions that are more accurate than the approach suggested in [11].

Example 9.1. $\varepsilon w^{\prime \prime}(s)+w^{\prime}(s-\delta)+w(s)=0$ with $w(s)=1, \forall-\delta \leq s \leq 0, w(1)=1$.

Example 9.2. $w^{\prime \prime}(s)+0.25 w^{\prime}(s-\delta)-w(s)=0$ with $w(s)=1, \forall-\delta \leq s \leq 0, w(1)=0$.

Example 9.3. $\varepsilon w^{\prime \prime}(s)-w^{\prime}(s-\delta)+w(s)=0$ with $w(s)=1, \forall-\delta \leq s \leq 0, w(1)=-1$. 
Table 6. The maximum errors in Example 9.1 for $\varepsilon=0.1$

\begin{tabular}{lccc}
\hline$N \downarrow$ & $\delta=0.03$ & $\delta=0.05$ & $\delta=0.08$ \\
\hline & & Results without fitting parameter \\
100 & $7.3234 \mathrm{e}-004$ & $9.8171 \mathrm{e}-004$ & \\
200 & $1.8325 \mathrm{e}-004$ & $2.4596 \mathrm{e}-004$ & $1.5000 \mathrm{e}-003$ \\
300 & $8.1441 \mathrm{e}-005$ & $1.0930 \mathrm{e}-004$ & $3.6947 \mathrm{e}-004$ \\
400 & $4.5805 \mathrm{e}-005$ & $6.1488 \mathrm{e}-005$ & $1.6414 \mathrm{e}-004$ \\
500 & $2.9318 \mathrm{e}-005$ & $3.9352 \mathrm{e}-005$ & $9.2331 \mathrm{e}-005$ \\
& & & $5.9087 \mathrm{e}-005$ \\
& & Results with fitting parameter \\
100 & $3.9018 \mathrm{e}-004$ & $5.3208 \mathrm{e}-004$ & \\
200 & $9.7620 \mathrm{e}-005$ & $1.3311 \mathrm{e}-004$ & $6.7428 \mathrm{e}-004$ \\
300 & $4.3393 \mathrm{e}-005$ & $5.9170 \mathrm{e}-005$ & $1.6872 \mathrm{e}-004$ \\
400 & $2.4410 \mathrm{e}-005$ & $3.3285 \mathrm{e}-005$ & $7.5003 \mathrm{e}-005$ \\
500 & $1.5623 \mathrm{e}-005$ & $2.1303 \mathrm{e}-005$ & $4.2190 \mathrm{e}-005$ \\
& & & $2.7002 \mathrm{e}-005$ \\
& & Results in [11] & \\
100 & $2.0790 \mathrm{e}-003$ & $5.3420 \mathrm{e}-003$ & $38340 \mathrm{e}-002$ \\
200 & $1.1020 \mathrm{e}-003$ & $3.2160 \mathrm{e}-003$ & $2.1830 \mathrm{e}-002$ \\
300 & $7.4900 \mathrm{e}-004$ & $2.2860 \mathrm{e}-003$ & $1.5200 \mathrm{e}-002$ \\
400 & $5.6800 \mathrm{e}-004$ & $1.7700 \mathrm{e}-003$ & $1.1650 \mathrm{e}-002$ \\
500 & $4.5700 \mathrm{e}-004$ & $1.4440 \mathrm{e}-003$ & $9.4430 \mathrm{e}-003$ \\
& & & \\
\hline
\end{tabular}

Table 7. The maximum errors in Example 9.1 for $\varepsilon=0.01$

\begin{tabular}{|c|c|c|c|c|}
\hline$N$ & $\delta=1 \times \varepsilon$ & $\delta=2 \times \varepsilon$ & $\delta=3 \times \varepsilon$ & $\delta=4 \times \varepsilon$ \\
\hline 500 & $2.6000 \mathrm{e}-003$ & $2.3000 \mathrm{e}-003$ & $2.1000 \mathrm{e}-003$ & $8.7507 \mathrm{e}-004$ \\
1000 & $6.4510 \mathrm{e}-004$ & $5.6801 \mathrm{e}-004$ & $5.2548 \mathrm{e}-004$ & $2.1969 \mathrm{e}-004$ \\
1500 & $2.8690 \mathrm{e}-004$ & $2.5258 \mathrm{e}-004$ & $2.3366 \mathrm{e}-004$ & $9.7715 \mathrm{e}-005$ \\
2000 & $1.6144 \mathrm{e}-004$ & $1.4210 \mathrm{e}-004$ & $1.3146 \mathrm{e}-004$ & $5.4979 \mathrm{e}-005$ \\
2500 & $1.0333 \mathrm{e}-004$ & $9.0955 \mathrm{e}-005$ & $8.4139 \mathrm{e}-005$ & $3.5191 \mathrm{e}-005$ \\
3000 & $7.1763 \mathrm{e}-005$ & $6.3166 \mathrm{e}-005$ & $5.8432 \mathrm{e}-005$ & $2.4440 \mathrm{e}-005$ \\
\hline
\end{tabular}


Table 8. The maximum errors in Example 9.2 for $\delta=0.03$

\begin{tabular}{|c|c|c|c|}
\hline$\varepsilon \downarrow$ & $N=100$ & $N=200$ & $N=400$ \\
\hline \multicolumn{4}{|c|}{ Results without fitting parameter } \\
\hline $2^{-1}$ & $7.2602 \mathrm{e}-007$ & $1.8150 \mathrm{e}-007$ & $4.5374 \mathrm{e}-008$ \\
\hline $2^{-2}$ & $2.9528 \mathrm{e}-006$ & $7.3816 \mathrm{e}-007$ & $1.8454 \mathrm{e}-007$ \\
\hline $2^{-3}$ & $1.1415 \mathrm{e}-005$ & $2.8533 \mathrm{e}-006$ & $7.1329 \mathrm{e}-007$ \\
\hline $2^{-4}$ & $4.3543 \mathrm{e}-005$ & $1.0882 \mathrm{e}-005$ & $2.7204 \mathrm{e}-006$ \\
\hline $2^{-5}$ & $1.8681 \mathrm{e}-004$ & $4.6707 \mathrm{e}-005$ & $1.1676 \mathrm{e}-005$ \\
\hline $2^{-6}$ & $1.0000 \mathrm{e}-003$ & $2.5198 \mathrm{e}-004$ & $6.3032 \mathrm{e}-005$ \\
\hline $2^{-7}$ & $6.5000 \mathrm{e}-003$ & $1.7000 \mathrm{e}-003$ & $4.1298 \mathrm{e}-004$ \\
\hline \multicolumn{4}{|c|}{ Results with fitting parameter } \\
\hline $2^{-1}$ & $5.0506 \mathrm{e}-007$ & $1.2627 \mathrm{e}-007$ & $4.5374 \mathrm{e}-008$ \\
\hline $2^{-2}$ & $1.6053 \mathrm{e}-006$ & $4.0133 \mathrm{e}-007$ & $1.8454 \mathrm{e}-007$ \\
\hline $2^{-3}$ & $4.2599 \mathrm{e}-006$ & $1.0650 \mathrm{e}-006$ & $7.1329 \mathrm{e}-007$ \\
\hline $2^{-4}$ & $2.3539 \mathrm{e}-005$ & $5.8863 \mathrm{e}-006$ & $2.7204 \mathrm{e}-006$ \\
\hline $2^{-5}$ & $1.3675 \mathrm{e}-004$ & $3.4211 \mathrm{e}-005$ & $1.1676 \mathrm{e}-005$ \\
\hline $2^{-6}$ & $7.3094 \mathrm{e}-004$ & $1.8316 \mathrm{e}-004$ & $6.3032 \mathrm{e}-005$ \\
\hline $2^{-7}$ & $3.3000 \mathrm{e}-003$ & $8.2563 \mathrm{e}-004$ & $4.1298 \mathrm{e}-004$ \\
\hline \multicolumn{4}{|c|}{ Results in [11] } \\
\hline $2^{-1}$ & $2.0800 \mathrm{e}-004$ & $1.0400 \mathrm{e}-004$ & $5.2000 \mathrm{e}-005$ \\
\hline $2^{-2}$ & $6.1200 \mathrm{e}-004$ & $3.0700 \mathrm{e}-004$ & $1.5400 \mathrm{e}-004$ \\
\hline $2^{-3}$ & $1.6340 \mathrm{e}-003$ & $8.2600 \mathrm{e}-004$ & $4.1500 \mathrm{e}-004$ \\
\hline $2^{-4}$ & $4.2470 \mathrm{e}-003$ & $2.1770 \mathrm{e}-003$ & $1.1020 \mathrm{e}-003$ \\
\hline $2^{-5}$ & $1.1675 \mathrm{e}-002$ & $6.1590 \mathrm{e}-003$ & $3.1660 \mathrm{e}-003$ \\
\hline $2^{-6}$ & $3.3680 \mathrm{e}-002$ & $1.8811 \mathrm{e}-002$ & $9.9520 \mathrm{e}-003$ \\
\hline $2^{-7}$ & $9.7727 \mathrm{e}-002$ & $5.9836 \mathrm{e}-002$ & $3.3166 \mathrm{e}-002$ \\
\hline
\end{tabular}

Table 9. The maximum errors in Example 9.2 for $\varepsilon=0.01$

\begin{tabular}{|c|c|c|c|c|}
\hline$N$ & $\delta=1 \times \varepsilon$ & $\delta=2 \times \varepsilon$ & $\delta=3 \times \varepsilon$ & $\delta=4 \times \varepsilon$ \\
\hline 500 & $5.4605 \mathrm{e}-005$ & $7.4563 \mathrm{e}-005$ & $7.9524 \mathrm{e}-005$ & $7.7839 \mathrm{e}-005$ \\
1000 & $1.3655 \mathrm{e}-005$ & $1.8645 \mathrm{e}-005$ & $1.9885 \mathrm{e}-005$ & $1.9464 \mathrm{e}-005$ \\
1500 & $6.0690 \mathrm{e}-006$ & $8.2870 \mathrm{e}-006$ & $8.8382 \mathrm{e}-006$ & $8.6510 \mathrm{e}-006$ \\
2000 & $3.4138 \mathrm{e}-006$ & $4.6615 \mathrm{e}-006$ & $4.9716 \mathrm{e}-006$ & $4.8662 \mathrm{e}-006$ \\
2500 & $2.1849 \mathrm{e}-006$ & $2.9834 \mathrm{e}-006$ & $3.1818 \mathrm{e}-006$ & $3.1144 \mathrm{e}-006$ \\
3000 & $1.5173 \mathrm{e}-006$ & $2.0718 \mathrm{e}-006$ & $2.2096 \mathrm{e}-006$ & $2.1628 \mathrm{e}-006$ \\
\hline
\end{tabular}


Table 10. The maximum error in Example 9.3 for $\varepsilon=0.1$

\begin{tabular}{cccc}
\hline$N \downarrow$ & $\delta=0.03$ & $\delta=0.05$ & $\delta=0.08$ \\
\hline & \multicolumn{2}{c}{ Results without fitting parameter } \\
100 & $4.7497 \mathrm{e}-004$ & $3.4420 \mathrm{e}-004$ & \\
200 & $1.1871 \mathrm{e}-004$ & $8.6029 \mathrm{e}-005$ & $2.3347 \mathrm{e}-004$ \\
300 & $5.2762 \mathrm{e}-005$ & $3.8234 \mathrm{e}-005$ & $5.8379 \mathrm{e}-005$ \\
400 & $2.9678 \mathrm{e}-005$ & $2.1506 \mathrm{e}-005$ & $2.5945 \mathrm{e}-005$ \\
500 & $1.8993 \mathrm{e}-005$ & $1.3764 \mathrm{e}-005$ & $1.4594 \mathrm{e}-005$ \\
& & Results with fitting parameter \\
100 & $5.3643 \mathrm{e}-004$ & $5.9925 \mathrm{e}-004$ & $9.3403 \mathrm{e}-006$ \\
200 & $1.3414 \mathrm{e}-004$ & $1.4988 \mathrm{e}-004$ & $5.3581 \mathrm{e}-004$ \\
300 & $5.9625 \mathrm{e}-005$ & $6.6620 \mathrm{e}-005$ & $1.3405 \mathrm{e}-004$ \\
400 & $3.3540 \mathrm{e}-005$ & $3.7475 \mathrm{e}-005$ & $5.9586 \mathrm{e}-005$ \\
500 & $2.1465 \mathrm{e}-005$ & $2.3985 \mathrm{e}-005$ & $3.3518 \mathrm{e}-005$ \\
& & $2.1452 \mathrm{e}-005$ \\
100 & $7.6000 \mathrm{e}-004$ & $1.1050 \mathrm{e}-003$ & \\
200 & $3.8000 \mathrm{e}-004$ & $5.5200 \mathrm{e}-004$ & $1.4140 \mathrm{e}-003$ \\
300 & $2.5300 \mathrm{e}-004$ & $3.6800 \mathrm{e}-004$ & $7.0700 \mathrm{e}-004$ \\
400 & $1.9000 \mathrm{e}-004$ & $2.7600 \mathrm{e}-004$ & $4.7200 \mathrm{e}-004$ \\
500 & $1.5200 \mathrm{e}-004$ & $2.2100 \mathrm{e}-004$ & $3.5400 \mathrm{e}-004$ \\
& & & $2.8300 \mathrm{e}-004$
\end{tabular}

Table 11. The maximum error in Example 9.3 for $\varepsilon=0.01$

\begin{tabular}{|c|l|l|l|l|}
\hline$N$ & $\delta=1 \times \varepsilon$ & $\delta=2 \times \varepsilon$ & $\delta=3 \times \varepsilon$ & $\delta=4 \times \varepsilon$ \\
\hline 500 & $7.1033 \mathrm{e}-004$ & $3.4103 \mathrm{e}-004$ & $2.0886 \mathrm{e}-004$ & $1.7059 \mathrm{e}-004$ \\
1000 & $1.7750 \mathrm{e}-004$ & $8.5246 \mathrm{e}-005$ & $5.2237 \mathrm{e}-005$ & $4.2912 \mathrm{e}-005$ \\
1500 & $7.8884 \mathrm{e}-005$ & $3.7886 \mathrm{e}-005$ & $2.3216 \mathrm{e}-005$ & $1.9094 \mathrm{e}-005$ \\
2000 & $4.4371 \mathrm{e}-005$ & $2.1311 \mathrm{e}-005$ & $1.3059 \mathrm{e}-005$ & $1.0745 \mathrm{e}-005$ \\
2500 & $2.8397 \mathrm{e}-005$ & $1.3639 \mathrm{e}-005$ & $8.3579 \mathrm{e}-006$ & $6.8781 \mathrm{e}-006$ \\
3000 & $1.9720 \mathrm{e}-005$ & $9.4714 \mathrm{e}-006$ & $5.8040 \mathrm{e}-006$ & $4.7770 \mathrm{e}-006$ \\
\hline
\end{tabular}




\section{Conclusions}

Numerical treatment of second-order linear convention-diffusion equations with a small delay in the convention term having layer behaviour is considered. A finite difference scheme is constructed using the non-polynomial spline and its first-order derivative continuity condition at the common node. If the delay $\delta=o(\varepsilon)$, the term containing the delay is expanded in Taylor's series and a fitted difference scheme is constructed for a layer at the left-end and rightend. The method is analyzed for convergence. For the Examples 6.1-6.5, Tables 1-5 shows the maximum absolute errors in the solutions. Comparing the computed errors with the results suggested in $[12,23,24]$, it was found that the proposed method yielded accurate results relative to the approaches suggested in $[12,23,24]$.

When the delay parameter $\delta(\varepsilon)$ is of order $O(\varepsilon)$, the behaviour of the layer can alter its nature and even be demolished or the solution displays oscillatory behaviour. In this case, a special mesh is applied so that, the term having delay parameter lies on the mesh points after the discretization and then the non-polynomial method is applied. Tables 6-11 of the numerical examples show the maximum absolute errors. Figures $1-8$ demonstrate the solutions of the examples for different values of the delay parameter. From Figure 1, Figure 3, Figure 5 it is noticed that, when the value of delay is greater than the perturbation, the solution involves oscillations. Further, if we increase the $\delta$ further, the oscillations which are restricted in the layer region are extend over whole domain and even move from one side to another.

To deal with these oscillations in solutions, we tried a new scheme with the use of the special type mesh introduced in [11] by adding a fitting parameter in the non-polynomial spline method. Examples 9.1-9.3 are considered, and the maximum absolute error was determined using the principle of double mesh $E^{N}=\max _{0 \leq i \leq N}\left|w_{i}^{N}-w_{2 i}^{2 N}\right|$. In Figures 2, 4, 6 of the computed solution, we showed the graphs with the fitting parameter for different values of $\delta=O(\varepsilon)$ and compared to the graphs without fitting factor. It is noticed that oscillations are regulated in solutions and layer behaviour is preserved, while the layer behaviour of the solution is preserved in the case of the layer at right-end (Figure 7 and 8), although the delay is of $O(\varepsilon)$. For the Examples 9.1-9.3, the maximum absolute errors with $\delta=O(\varepsilon)$, are tabulated in Tables $7,9,11$. It is observed from the tabulated results that the maximum absolute errors are also decreasing as the mesh size $h$ decreases.

Based on the graphs of the solutions in Fig 1-8, it has also been concluded that the method proposed with a fitting parameter had a great advantage in regulating the oscillation in the solutions of the linear singularly perturbed differential delay equations. 


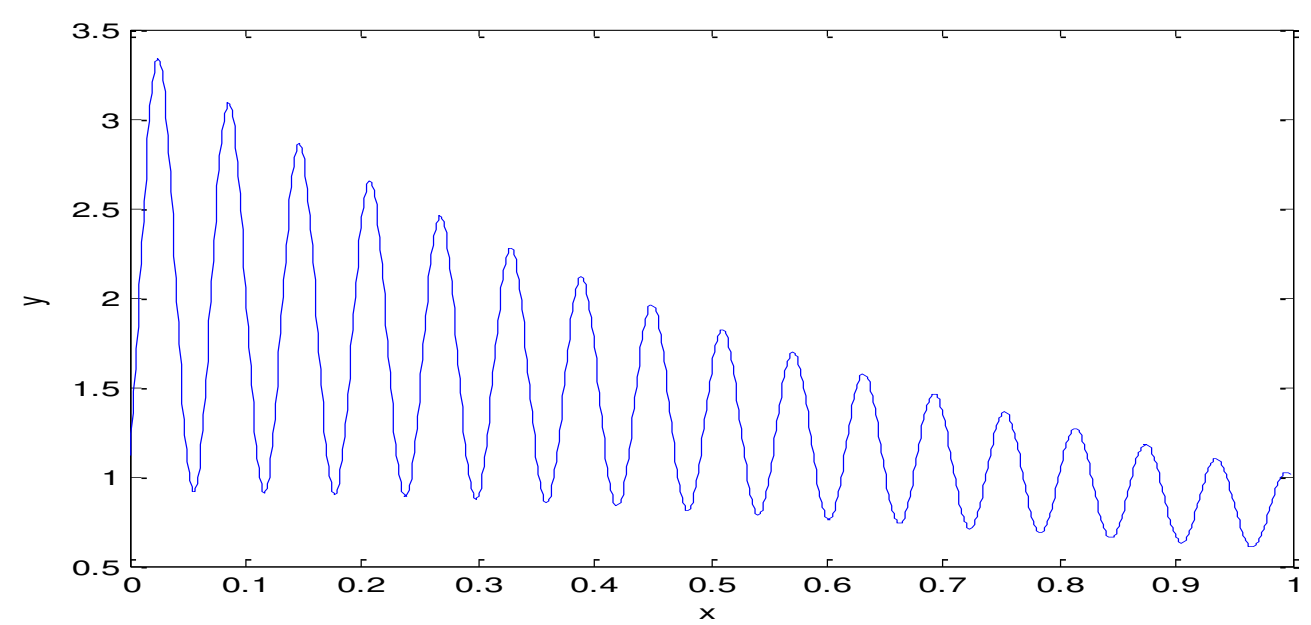

Figure 1. Numerical solution in Example 9.1 for $\varepsilon=0.01$ and $\delta=1.5 \varepsilon$ without fitting parameter.

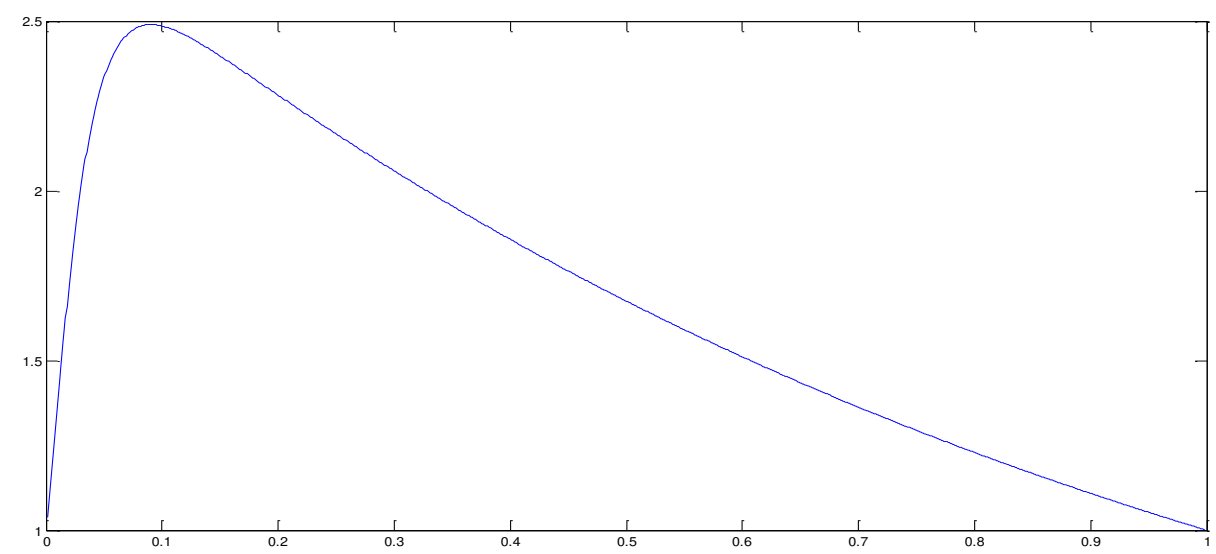

Figure 2. Numerical solution in Example 9.1 for $\varepsilon=0.01$ and $\delta=1.5 \varepsilon$ with fitting parameter.

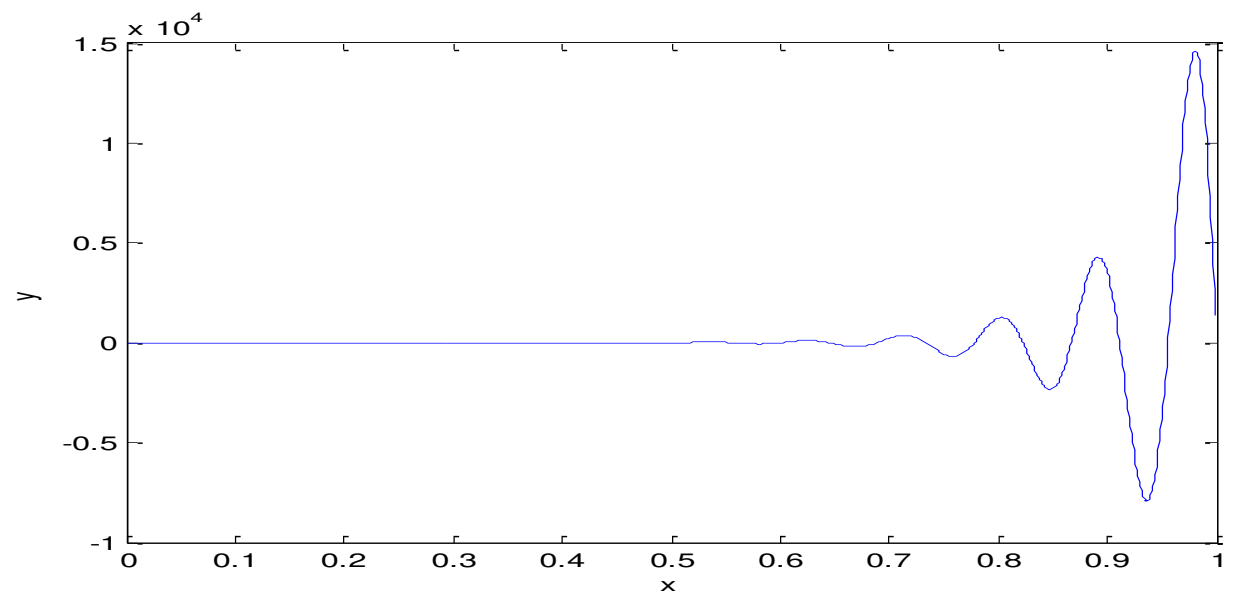

Figure 3. Numerical solution in Example 9.1 for $\varepsilon=0.01$ and $\delta=2.5 \varepsilon$ without fitting parameter. 


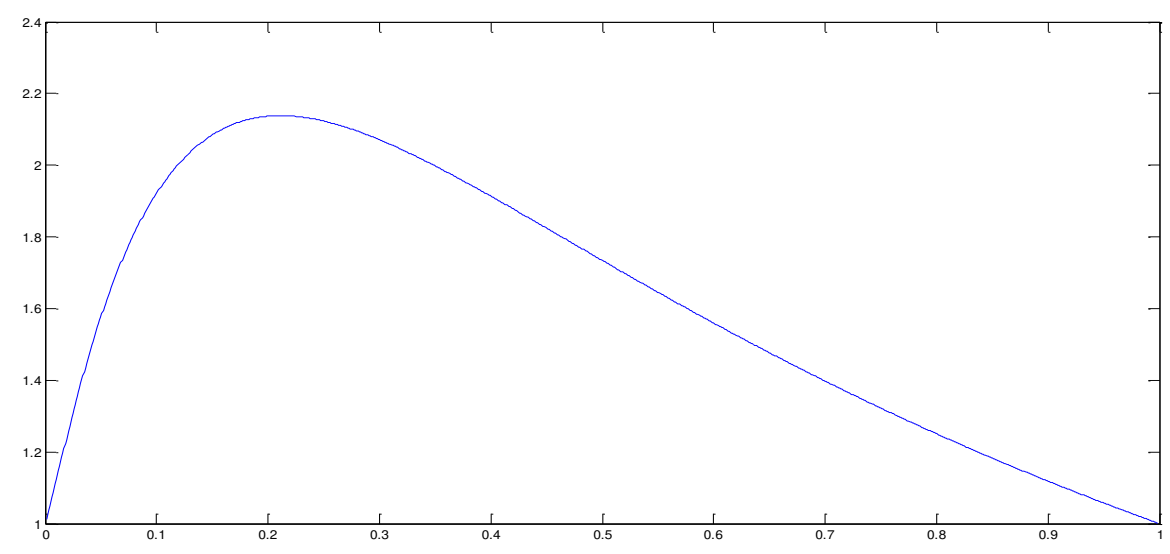

Figure 4. Numerical solution in Example 9.1 for $\varepsilon=0.01$ and $\delta=2.5 \varepsilon$ with fitting parameter.

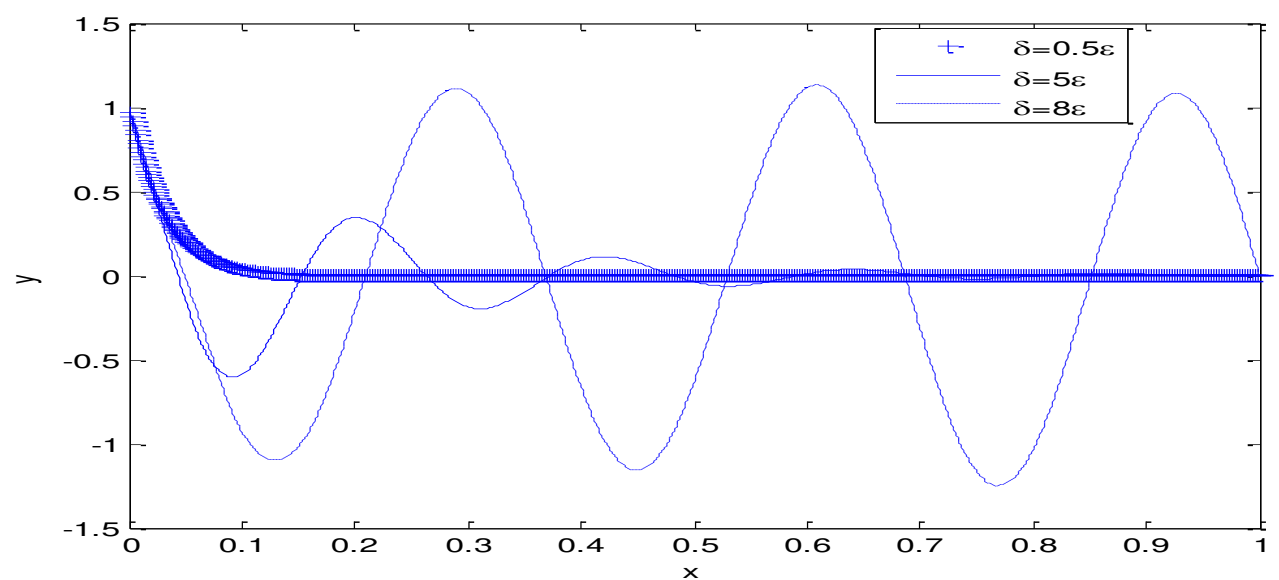

Figure 5. Numerical solution in Example 9.2 for $\varepsilon=0.01$ with different values of $\delta$ without fitting parameter

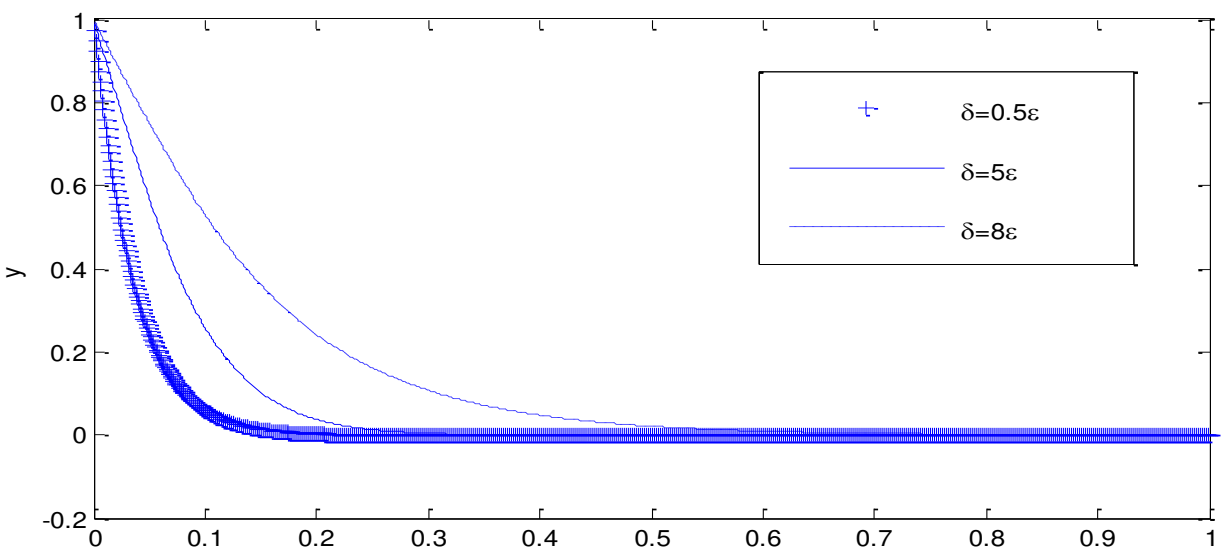

Figure 6. Numerical solution in Example 9.2 for $\varepsilon=0.01$ with different values of $\delta$ with fitting parameter 


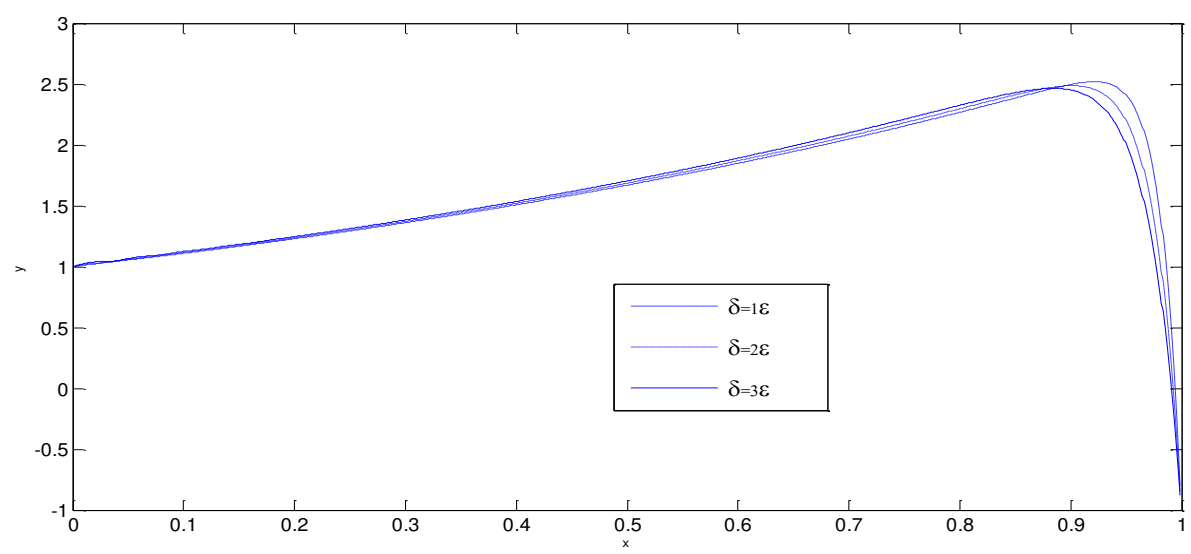

Figure 7. Numerical solution in Example 9.3 for $\varepsilon=0.01$ with different values of $\delta$ without fitting parameter

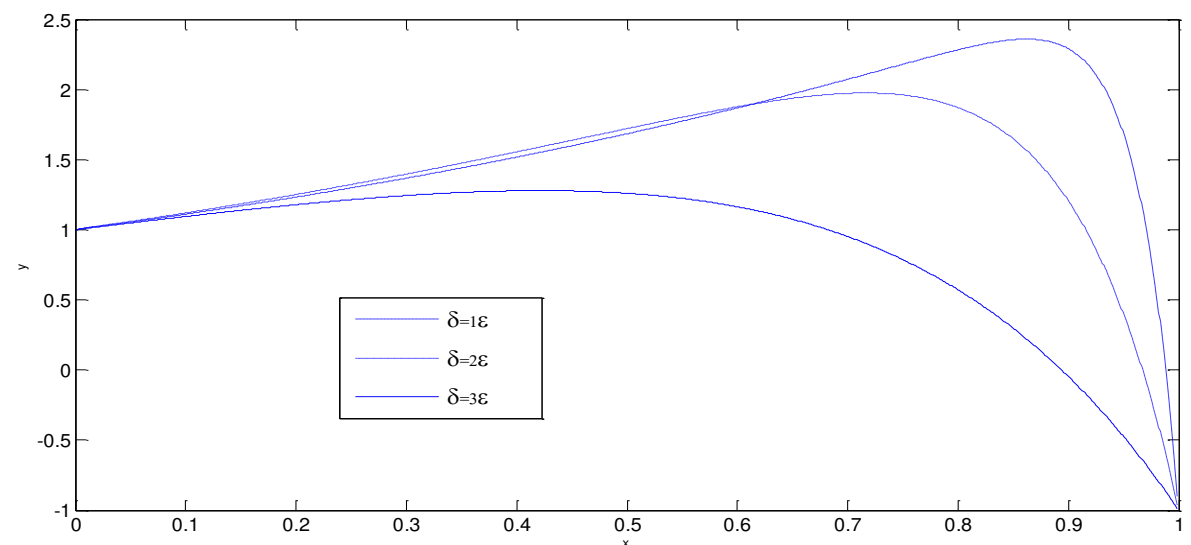

Figure 8. Numerical solution in Example 9.3 for $\varepsilon=0.01$ with different values of $\delta$ with fitting parameter

\section{Compliance with ethical standards}

Conflict of interest The authors declares that they have no conflict of interest.

Ethical approval This article does not contain any studies with human participants or animals performed by any of the authors. 


\section{References}

[1] Bellman R, Cooke K (1963) Differential-Difference Equations, Academic Press, New York.

[2] Bestehornand York M, Grigorieva EV (2004) Formation and propagation of localized states in extended systems. Ann Phys 13: 423-431.

[3] Derstine MW, Gibbs HM, Hopf FA, Kaplan DL (1982) Bifurcation gap in a hybrid optical system. Phys Rev A 26: 3720-3722.

[4] Doolan EP, Miller JJH, Schilders WHA (1980) Uniform Numerical Methods for Problems with Initial and Boundary Layers. Boole Press, Dublin.

[5] Driver RD (1977) Ordinary and Delay Differential Equations. Belin-Heidelberg, New York, Springer.

[6] El'sgol'ts LE, Norkin SB (1973) Introduction to the theory and application of differential equations with deviating arguments. Academic Press.

[7] Glizer VY (2000) Asymptotic solution of a boundary-value problem for linear singularly perturbed functional differential equations arising in optical control theory. J Optim Theory Appl 106: 309-335.

[8] Jalil Rashidinia And Reza Jalilian (2010) Spline solution of two-point boundary value problems. Applied and Computational Mathematics, 9: 258-266.

[9] Kadalbajoo MK, Sharma KK (2004) Numerical analysis of singularly perturbed delay differential equations with layer behavior. Appl Math and Compu 157: 11-28.

[10] Kadalbajoo MK, Sharma KK (2005) Numerical treatment of boundary value problems for second order singularly perturbed delay differential equations. J Comput and Appl Math 24: 151-172.

[11] Kadalbajoo MK, Sharma KK (2008) A Numerical method based on finite difference for boundary value problems for singularly perturbed delay differential equations. Appl Math and Comput 197: 692-707.

[12] Kadalbajoo MK, Kumar D (2010) A computational method for singularly perturbed nonlinear differential-difference equations with small shift. Applied Mathematical Modelling 34: 2584-2596.

[13] Kokotovic PV, Khalil HK, O’Reilly J (1986) Singular perturbation methods in control analysis and design. Academic Press, New York.

[14] Kuang Y (1993) Delay Differential Equations with Applications in Population Dynamics. Academic Press, New York. 
[15] Lange CG, Miura RM (1994) Singular perturbation analysis of boundary-value problems for differential-difference equations. v. small shifts with layer behavior. SIAM J Appl Math 54: 249-272.

[16] Lange CG, Miura RM (1994) Singular perturbation analysis of boundary-value problems for differential-difference equations. vi. Small shifts with rapid oscillations. SIAM J Appl Math 54: 273-283.

[17] Lasota A, Wazewska M (1976) Mathematical models of the red blood cell system. Mat Stos 6: $25-40$.

[18] Mackey MC, Glass L (1977) Oscillation and chaos in physiological control systems. Science, 197: 287-289.

[19] Martin A, Raun S (2001) Predetor-prey models with delay and prey harvesting. J Math Bio 43: 247-267.

[20] Miller JJH, O'Riordan RE, Shishkin GI (1996) Fitted numerical methods for singular perturbation problems. World Scientific, Singapore.

[21] O’Malley RE (1974) Introduction to Singular Perturbations, Academic Press, New York.

[22] Rashidinia J, Mohammadi R, Moatamedoshariati SH (2010) Quintic Spline Methods for the Solution of Singularly Perturbed Boundary-Value Problems. International Journal for Computational Methods in Engineering Science and Mechanics 11: 247-257.

[23] Ravikanth ASV, Murali P (2017) Numerical treatment for a singularly perturbed convection delayed dominated diffusion Equation via tension splines. Inter $\mathrm{J}$ of Pure and Appl Math 113: 110-118.

[24] Reddy YN, Soujanya GBSL, Phaneendra K (2012) Numerical Integration Method for Singularly Perturbed Delay Differential Equations. Inter J of Appl Sci and Engin 10: 249261.

[25] Smith H (2010) An Introduction to Delay Differential Equations with Applications to the Life Sciences. Springer, Berlin.

[26] Stein RB (1967) Some models of neuronal variability. Biophys J 7: 37-68.

[27] Varga RS (1962) Matrix Iterative Analysis. Prentice-Hall, Englewood Cliffs, New Jersey.

[28] Young DM (1971) Iterative Solutions of Large Linear Systems. Academic press, New York. 